\title{
Timed Get Up and Go Test
}

National Cancer Institute

\section{Source}

National Cancer Institute. Timed Get Up and Go Test. NCI Thesaurus. Code C157021.

A test that measures the amount of time it takes a person to rise from a chair, walk to a designated point, and return to sitting. 